\title{
SPACES OF GEODESICS: PRODUCTS, COVERINGS, CONNECTEDNESS
}

\author{
John K. Beem \\ Robert J. Low \\ Department of Mathematics \\ University of Missouri \\ Columbia MO 65211 \\ USA \\ mathjkb@mizzou1.missouri.edu
Department of Mathematics
Coventry University
Coventry CV1 5FB
UK \\ roblow@coventry.ac.uk \\ Phillip E. Parker \\ Department of Mathematics \\ The Wichita State University \\ Wichita KS 67260-0033 \\ USA \\ pparker@twsuvm.uc.twsu.edu
}

January 14, 1995

MSC (1991): Primary 53C22; Secondary 53C50. 


\begin{abstract}
We continue our study of the space of geodesics of a manifold with linear connection. We obtain sufficient conditions for a product to have a space of geodesics which is a manifold. We investigate the relationship of the space of geodesics of a covering manifold to that of the base space. We obtain sufficient conditions for a space to be geodesically connected in terms of the topology of its space of geodesics. We find the space of geodesics of an $n$-dimensional Hadamard manifold is the same as that of $\mathbb{R}^{n}$.
\end{abstract}




\section{Introduction}

A topological space is locally Euclidean if and only if every point has a neighborhood homeomorphic to an open set in some $\mathbb{R}^{n}$. Such a space is sometimes called a manifold which is not Hausdorff. Here, we shall assume that locally Euclidean spaces are paracompact (not necessarily Hausdorff) and connected, and a manifold will be a Hausdorff locally Euclidean space. Note that the theory of differential structures (smoothness) does not depend on the Hausdorff condition, so that one may speak of smooth $\left(C^{\infty}\right)$ locally Euclidean spaces. We agree that all locally Euclidean spaces are smooth. Thus there is a tangent bundle $T E$, etc. Notations will be given explicitly only for manifolds, but may be used for locally Euclidean spaces whenever appropriate.

For a smooth manifold $M$ with tangent bundle $T M$, let $T^{\prime} M$ denote the reduced tangent bundle consisting of $T M$ less the zero section and let $P M$ denote the quotient space of $T^{\prime} M$ obtained by identifying proportional vectors at each $p \in M$. If $M$ is $n$-dimensional, then $P M$ has compact fibers diffeomorphic to the real projective space $\mathbb{P}^{n-1}$. We shall regard points in $P M$ as tangent lines to curves in $M$. There is also the double covering $S M$ of $P M$ in which the proportionality must be by positive numbers and the compact fibers are diffeomorphic to the sphere $S^{n-1}$.

When $M$ is provided with a linear connection, there are geodesics in $M$. We agree that all geodesics are to be inextendible (maximally extended). Define the space of (unoriented) geodesics $G(M)$ to be the quotient of PM obtained by identifying points that lie on the lift of a common geodesic. Elements of $G(M)$ may be regarded as equivalence classes $[\gamma]$ of parameterized geodesics $\gamma:(a, b) \longrightarrow M$. Similarly, there is the space of oriented geodesics $G^{+}(M)$, the quotient of $S M$. Observe that different connections on $M$ may yield topologically different spaces of geodesics.

One usually regards closed and periodic as synonymous for geodesics. However, it is possible for a null geodesic to retrace its path without the tangent vector returning to itself: since it has zero length, it need return only to a scalar multiple of itself. A linear connection need not be metric, and then this could happen with all the geodesics which retrace themselves. Thus we shall distinguish between closed geodesics in which the image is a closed curve and periodic geodesics in which the image of the natural lift to $T M$ is a closed curve. Observe that for spacelike and timelike geodesics of a pseudoriemannian metric tensor, closed and periodic are still synonymous. In particular, this is true for all geodesics of a Riemannian metric. 
This paper continues [3] and [8], and contains proofs of most of the results announced in [12]; proofs of the others will appear later.

Let $\left(M_{1}, \nabla_{1}\right)$ and $\left(M_{2}, \nabla_{2}\right)$ be two manifolds with linear connections. In Section 2 we consider the space of geodesics of the product $\left(M_{1} \times M_{2}, \nabla_{1} \times\right.$ $\left.\nabla_{2}\right)$. We find that if $G\left(M_{1} \times M_{2}\right)$ is locally Euclidean, then both $G\left(M_{1}\right)$ and $G\left(M_{2}\right)$ must be locally Euclidean. On the other hand, if both $G\left(M_{1}\right)$ and $G\left(M_{2}\right)$ are locally Euclidean, then $G\left(M_{1} \times M_{2}\right)$ is locally Euclidean if and only if both $\left(M_{1}, \nabla_{1}\right)$ and $\left(M_{2}, \nabla_{2}\right)$ fail to have any closed geodesics. In particular, one may easily construct examples with both $G\left(M_{1}\right)$ and $G\left(M_{2}\right)$ manifolds, but $G\left(M_{1} \times M_{2}\right)$ not a manifold. Stronger results may be obtained when the geodesics of $\left(M_{1} \times M_{2}, \nabla_{1} \times \nabla_{2}\right)$ satisfy the nonreturning property. Under this assumption, $G\left(M_{1} \times M_{2}\right)$ is a manifold if and only if both $G\left(M_{1}\right)$ and $G\left(M_{2}\right)$ are manifolds.

In Section 3 we consider smooth coverings with projections that preserve geodesics. If $p: \widetilde{M} \rightarrow M$ is such a covering, then there is an induced map $\hat{p}: G(\widetilde{M}) \rightarrow G(M)$ and this induced map commutes with the natural projections $T^{\prime}(M) \rightarrow G(M)$ and $T^{\prime}(\widetilde{M}) \rightarrow G(\widetilde{M})$. In general $G(\widetilde{M})$ may fail to be a manifold when $G(M)$ is a manifold. However, if $(M, \nabla)$ is nonreturning then $G(\widetilde{M})$ will be a manifold when $G(M)$ is a manifold.

The Hopf-Rinow Theorem guarantees that if $(M, g)$ is a complete Riemannian (positive definite) manifold, then each pair of points may be joined by at least one geodesic. Thus, one says that a complete Riemannian manifold is geodesically connected. In spaces which are not positive definite, there is no Hopf-Rinow Theorem and the problem of deciding if each pair of points may be joined by some geodesic can be quite difficult. In Section 4 we consider the problem of geodesic connectedness. We find that $(M, \nabla)$ is geodesically connected if it is unitrace and $G(M)$ is Hausdorff. We define sky bundles and find that if one has a sky bundle with the top Stiefel-Whitney class nontrivial, then one may connect pairs of points with geodesic segments. This yields a new approach to the problem of deciding when a manifold with a linear connection is geodesically connected. In Section 4, we also show that for Hadamard manifolds the geodesic space is the same as that of $\mathbb{R}^{n}$.

\section{Products}

We begin by recalling some definitions and results from [2, 3] and [12]. From [3] we need 
Definition 2.1 $(M, \nabla)$ is unitrace if and only if for each $p \in M$ and each neighborhood $V$ of $p$ there is a simple convex neighborhood $U$ of $p$ with $U \subseteq V$ such that any geodesic which enters $U$ either leaves and never returns or retraces the same path every time it does return. If each point has arbitrarily small neighborhoods such that no geodesic ever returns, we say $(M, \nabla)$ is nonreturning.

Observe that nonreturning implies unitrace implies the geodesic foliation of $P M$ is regular in the sense of Palais [11]. As in [12], we make the

Definition $2.2(M, \nabla)$ is said to be geodesically regular if and only if the geodesic foliation of $P M$ is regular.

This means that each point in $P M$ has a neighborhood which is intersected by each lifted geodesic at most once. In particular, this implies that each point of $M$ has a neighborhood which every geodesic image crosses at most finitely many times. This is not true on the Misner cylinder $\mathcal{M}$ [5, p.177ff]. When the connection is flat, the crossings cannot be parallel and arbitrarily close. This is not true on the flat Möbius band $\mathfrak{M}$. From [11, p. 19] we obtain [12, Theorem 3.15]

Theorem $2.3(M, \nabla)$ is geodesically regular if and only if $G(M)$ is locally Euclidean.

Finally we recall [2, 3]

Definition 2.4 We say that $(M, \nabla)$ is disprisoning if and only if each end of each geodesic eventually leaves each compact set. $(M, \nabla)$ is $\nabla$-pseudoconvex if and only if for every compact set $K$ there is a compact set $K^{\prime}$ such that every geodesic segment with both endpoints in $K$ lies entirely in $K^{\prime}$.

One regards pseudoconvexity as a sort of interior completeness condition.

Now let $\left(M_{1}, \nabla_{1}\right)$ and $\left(M_{2}, \nabla_{2}\right)$ be manifolds with linear connections. In general, the space of geodesics of the product cannot readily be determined from those of the factors. Some properties, however, are reasonably well shared by a product and its factors.

Proposition 2.5 Let $\left(M_{1}, \nabla_{1}\right)$ and $\left(M_{2}, \nabla_{2}\right)$ be geodesically regular. The product $\left(M_{1} \times M_{2}, \nabla_{1} \times \nabla_{2}\right)$ is geodesically regular if and only if neither factor has a closed geodesic. 
Proof: Assume one factor, say $M_{1}$, has a closed geodesic $\gamma$ with $\gamma^{\prime}(0)=$ $c \gamma^{\prime}(a)$. Let $\beta$ be any geodesic of $M_{2}$ with 0 in the domain of $\beta$. Set $\lambda(\varepsilon, t)=(\gamma(t), \beta(\varepsilon t))$. For each fixed $\varepsilon$, one has $\lambda(\varepsilon, t)$ a geodesic of $M_{1} \times M_{2}$ and $\lambda(\varepsilon, a) \rightarrow(\gamma(0), \beta(0))=(\gamma(a), \beta(0))$ as $\varepsilon \rightarrow 0$. Furthermore, $\lambda^{\prime}(\varepsilon, 0)$ and $\lambda^{\prime}(\varepsilon, a)$ converge to $\left(\gamma^{\prime}(0), 0\right)$ and $\left(\gamma^{\prime}(a), 0\right)$, respectively, as $\varepsilon \rightarrow 0$. It follows that each neighborhood of the point in $P\left(M_{1} \times M_{2}\right)$ corresponding to $\left(\gamma^{\prime}(0), 0\right)$ is intersected more than once by the curve of tangent lines corresponding to $\lambda^{\prime}(\varepsilon, t)$ for all sufficiently small $\varepsilon \neq 0$. Thus the product is not geodesically regular.

Conversely, if $M_{1} \times M_{2}$ is not geodesically regular, then there exists $v=\left(v_{1}, v_{2}\right) \in T_{x}\left(M_{1} \times M_{2}\right) \cong T_{x_{1}} M_{1} \times T_{x_{2}} M_{2}$ where geodesic regularity fails. Let $U$ be any simple convex normal neighborhood of $x$. Then there is a sequence of geodesics $\lambda_{n}=\left(\gamma_{n}, \beta_{n}\right)$ such that $\lambda_{n}^{\prime}(0) \rightarrow v$ and the tangent lines containing $\lambda_{n}^{\prime}\left(t_{n}\right)$ converge to the tangent line containing $v$ for some sequence $\left\{t_{n}\right\}$ where $\lambda_{n}$ leaves $U$ between 0 and $t_{n}$. Let $U_{i}$ be the projection of $U$ onto $M_{i}$ for $i=1,2$. Without loss of generality, we may as well assume that the geodesics $\gamma_{n}$ of $M_{1}$ leave $U_{1}$ after $t=0$ and return before $t=t_{n}$ with tangent lines converging to that containing $v_{1}$. Since $M_{1}$ is geodesically regular, it follows that $\gamma_{n}$ must retrace its image in $U_{1}$ for all large $n$. Therefore $M_{1}$ has a closed geodesic.

Using Theorem 2.3, we obtain

Theorem 2.6 Let $G\left(M_{1}\right)$ and $G\left(M_{2}\right)$ be locally Euclidean. The space of geodesics of the product $G\left(M_{1} \times M_{2}\right)$ is locally Euclidean if and only if neither factor has a closed geodesic.

The space of geodesics of the product need not be Hausdorff either, even if that of each factor is. A simple example is the standard pseudoeuclidean $\mathbb{R}^{n} \times S^{k}, n, k>0$.

Proposition $2.7\left(M_{1} \times M_{2}, \nabla_{1} \times \nabla_{2}\right)$ is nonreturning if and only if $\left(M_{1} \times\right.$ $\left.M_{2}, \nabla_{1} \times \nabla_{2}\right)$ is unitrace if and only if $\left(M_{1}, \nabla_{1}\right)$ and $\left(M_{2}, \nabla_{2}\right)$ are both nonreturning.

Proof: Since geodesics of the product are ordered pairs of geodesics of the factors, it follows easily that the product is nonreturning if and only if both factors are.

Clearly, if the product is nonreturning then it is unitrace. It only remains to show that if the product is unitrace, then it is nonreturning. To this 
end, assume that the product has a geodesic $\gamma=\left(\gamma_{1}, \gamma_{2}\right)$ that leaves a neighborhood $U$ of $\gamma(0)$ and later returns and retraces its image. At least one of $\gamma_{1}$ or $\gamma_{2}$ is nontrivial, so we may as well assume that $\gamma_{1}$ is nontrival. Then there is some $a$ and $c$ with $\gamma_{1}^{\prime}(0)=c \gamma_{1}^{\prime}(a)$. Let $\beta$ be any nontrivial geodesic of $\left(M_{2}, \nabla_{2}\right)$ and set $\lambda(\varepsilon, t)=\left(\gamma_{1}(t), \beta(\varepsilon t)\right)$. Letting $\varepsilon \rightarrow 0$ as in the first part of the proof of Proposition 2.5, we obtain a failure of geodesic regularity. But the product is unitrace, hence geodesically regular; contradiction.

Theorem 2.8 If $G\left(M_{1} \times M_{2}\right)$ is locally Euclidean, then so are $G\left(M_{1}\right)$ and $G\left(M_{2}\right)$.

Proof: Using Theorem 2.3, we need only show that geodesic regularity of the product implies geodesic regularity of the factors. Fixing $\left(x_{1}, x_{2}\right) \in$ $M_{1} \times M_{2}$, the result follows easily from the identification of $M_{1}$ with the submanifold $M_{1} \times\left\{x_{2}\right\}$ of $M_{1} \times M_{2}$ and of $M_{2}$ with the corresponding $\left\{x_{1}\right\} \times M_{2}$.

Lemma $2.9\left(M_{1} \times M_{2}, \nabla_{1} \times \nabla_{2}\right)$ is pseudoconvex if and only if $\left(M_{1}, \nabla_{1}\right)$ and $\left(M_{2}, \nabla_{2}\right)$ both are.

Proof: Let $r_{1}$ and $r_{2}$ be the canonical projections onto the first and second factors, respectively, and let $\left(x_{1}, x_{2}\right)$ be a fixed point of the product.

First, assume that the product is pseudoconvex and let $K$ be a compact set in $M_{1}$. Then $K \times\left\{x_{2}\right\}$ is a compact subset of the product, hence there is a compact $H \subseteq M_{1} \times M_{2}$ such that any geodesic segment of $M_{1} \times M_{2}$ with endpoints in $K \times\left\{x_{2}\right\}$ must lie in $H$. If $\gamma:[a, b] \rightarrow M_{1}$ is a geodesic segment in $M_{1}$ with endpoints in $K$, then $\lambda=\left(\gamma, x_{2}\right)$ is a geodesic segment in $M_{1} \times M_{2}$ with endpoints in $K \times\left\{x_{2}\right\}$ and image in $H$. Consequently, $\gamma=r_{1} \circ \lambda$ lies in the compact set $r_{1}(H)$ for any such $\lambda$. It follows that $\left(M_{1}, \nabla_{1}\right)$ is pseudoconvex; the argument for $\left(M_{2}, \nabla_{2}\right)$ is similar.

Conversely, assume that the factors are pseudoconvex. Now let $K \subseteq$ $M_{1} \times M_{2}$ be compact and set $K_{1}=r_{1}(K)$ and $K_{2}=r_{2}(K)$. There are compact $H_{i} \subseteq M_{i}$ such that any geodesic segment of $M_{i}$ with endpoints in $K_{i}$ must lie in $H_{i}$. Now $H=H_{1} \times H_{2}$ is compact. Since geodesics of the product are ordered pairs of geodesics of the factors, it follows that any geodesic of $M_{1} \times M_{2}$ with endpoints in $K \subseteq K_{1} \times K_{2}$ must lie in $H$. Therefore the product is pseudoconvex.

Using this lemma and Corollary 5.6 of [3], we obtain 
Theorem 2.10 If $\left(M_{1} \times M_{2}, \nabla_{1} \times \nabla_{2}\right)$ is nonreturning, then these are equivalent:

1. $G\left(M_{1} \times M_{2}\right)$ is Hausdorff;

2. $G\left(M_{1} \times M_{2}\right)$ is a manifold;

3. $\left(M_{1} \times M_{2}, \nabla_{1} \times \nabla_{2}\right)$ is pseudoconvex;

4. $G\left(M_{1}\right)$ and $G\left(M_{2}\right)$ are Hausdorff;

5. $G\left(M_{1}\right)$ and $G\left(M_{2}\right)$ are manifolds;

6. $\left(M_{1}, \nabla_{1}\right)$ and $\left(M_{2}, \nabla_{2}\right)$ are pseudoconvex.

Combining Proposition 2.7 and Theorem 2.10, we obtain sufficient conditions for a product to have a space of geodesics which is a manifold.

Theorem 2.11 If $\left(M_{1}, \nabla_{1}\right)$ and $\left(M_{2}, \nabla_{2}\right)$ are each both nonreturning and pseudoconvex, then $G\left(M_{1} \times M_{2}\right)$ is a manifold.

The general problem to construct $G\left(M_{1} \times M_{2}\right)$ from $G\left(M_{1}\right)$ and $G\left(M_{2}\right)$ is complicated and somewhat difficult, and more work needs to be done here. We shall now construct $G(\mathbb{R} \times M)$ for $M$ with a linear connection.

Construction 2.12 Coordinatize $R \times M$ by $(s, x)$ where $s \in R$ and $x$ gives the coordinates of a point in $M$. We shall call $\partial_{s}$ the horizontal direction and refer to directions tangent to $M$ as vertical. Let $p$ be the natural projection to $M$, and identify $M$ with $\{0\} \times M$.

First, consider all the geodesics in $\mathbb{R} \times M$ that project to the nondegenerate geodesic in $\mathbb{R}$. All geodesics of this form must pass through some point $(0, x)$ for some $x$, and are completely specified by their tangent vector there. We normalize so that the projection to $\mathbb{R}$ has unit velocity. Then the nonvertical geodesics are given exactly by $T M$. Next, there are the vertical geodesics to consider. For each $s \in \mathbb{R}$, we obtain the whole of $G(M)$. Thus, as a set, we have $T M \sqcup \mathbb{R} \times G(M)$. The only remaining question is, what is the topology? This can be settled by seeing what sequences in $T M$ converge to which points (if any) in $\mathbb{R} \times G(M)$.

Recall that each point $(x, v)$ of $T M$ specifies the geodesic through $(0, x)$ with tangent $(1, v)$. Let $\left(x_{n}, v_{n}\right)$ be a sequence in $T M$; under what circumstances will this sequence, regarded as a set of geodesics in $\mathbb{R} \times M$, converge to some vertical geodesic? Denote the geodesic corresponding to $\left(x_{n}, v_{n}\right)$ by 
$\gamma_{n}$, where $\gamma(0)=\left(0, x_{n}\right)$, and $\dot{\gamma}_{n}=\left(1, v_{n}\right)$, and let $\gamma$ be the vertical geodesic with $\gamma(0)=(s, x)$ and $\dot{\gamma}_{n}=(0, v)$. Then $\gamma_{n} \rightarrow \gamma$ if and only if $p\left(\gamma_{n}\right) \rightarrow \gamma$, and the point of intersection of $\gamma_{n}$ with $\{s\} \times M$ converges to $(s, x)$.

This construction can be iterated to describe the set $G\left(\mathbb{R}^{k} \times M\right)$ for any standard pseudoeuclidean $\mathbb{R}^{k}$, but the details of the topology are rather complicated.

In the case of $\mathbb{R} \times M$, it is easy to see that if $M$ has a closed geodesic, then a sequence in $T M$ converging to one vertical copy of this geodesic will converge to all copies as for the classical cylinder. It is also easy to carry out this construction explicitly for the case where $M$ is $\mathbb{R}$ and obtain a Möbius band.

Example 2.13 First, consider all the nonvertical geodesics in $\mathbb{R} \times \mathbb{R}=$ $\{(x, y)\}$. These geodesics are completely described by $T \mathbb{R}$ via the $y$-intercept $(0, y)$ and the normalized velocity $(1, v)$. Now $T \mathbb{R}$ is another copy $\mathbb{R} \times \mathbb{R}=$ $\{(u, v)\}$. The point $(u, v)$ corresponds to the nonvertical geodesic with $y$ intercept $(0, u)$ and normalized velocity $(1, v)$.

Next we consider those sequences of nonvertical geodesics which converge to a vertical geodesic, in order to attach the boundary copy of $\mathbb{R}$ in the correct way. Any sequence of nonvertical geodesics through the point $(x, 0)$ in $\mathbb{R}_{x y}^{2}$ which converges to the vertical geodesic through $(x, 0)$ has a sequence of normalized velocities $\left(1, v_{n}\right)$ with $v_{n} \rightarrow \pm \infty$. This yields the sequence of points $\left(-x v_{n}, v_{n}\right)$ in $\mathbb{R}_{u v}^{2}$. Observe that these points lie along the line through the origin of slope $-1 / x$, interpreted as the $v$-axis when $x=0$.

Now shrink $\mathbb{R}_{u v}^{2}$ to the unit disc; e.g., map the point with polar coordinates $(r, \theta)$ to the point $(\tanh r, \theta)$. We see that we should attach one boundary point to each antipodal position pair except the pair $( \pm 1,0)$ at opposite ends of the $u$-axis. This recovers $G\left(\mathbb{R}^{2}\right)$ as the projective plane less a point: the open Möbius band.

If we consider instead the oriented geodesics of $\mathbb{R} \times \mathbb{R}$, we obtain two copies of the disc glued along the boundaries in the oriented way except at the points $( \pm 1,0)$ again. This yields $S^{2}$ less two antipodal points, diffeomorphic to $T S^{1}$. 


\section{Coverings}

Let $p: \widetilde{M} \rightarrow M$ be a smooth covering map; in particular, we assume that $p$ is a local diffeomorphism which evenly covers $M$. We have

Lemma 3.1 If $p$ is a such a covering map, then $p$ and the induced tangent map $p_{*}$ are open.

Definition 3.2 We say that $p:(\widetilde{M}, \widetilde{\nabla}) \rightarrow(M, \nabla)$ is a geodesic covering map if and only if $p$ takes the image of each geodesic in $(\widetilde{M}, \widetilde{\nabla})$ onto the image of a geodesic in $(M, \nabla)$.

Such maps preserving geodesics up to parameterization are called projective (cf. [6, 7] for older terminology).

Now suppose $p$ is a geodesic covering map. Let $\pi: T^{\prime} M \rightarrow G(M)$, and $\tilde{\pi}: T^{\prime} \widetilde{M} \rightarrow G(\widetilde{M})$ be the natural projections.

Proposition 3.3 There is an induced map $\hat{p}: G(\widetilde{M}) \rightarrow G(M)$ which is a continuous, open surjection (thus a quotient map) such that $\hat{p} \tilde{\pi}=\pi p_{*}$.

Proof: Fix $[\gamma] \in G(\widetilde{M})$ and pull back to $T^{\prime} \widetilde{M}$ by using $G=\tilde{\pi}^{-1}([\gamma])$. We shall show that if $u, v \in G$, then $\pi p_{*}(u)=\pi p_{*}(v)$, which will show that for each $[\gamma] \in G(\widetilde{M})$ we have a uniquely defined element $\hat{p}([\gamma])=\pi p_{*}(G)=$ $\pi p_{*}(u)=\pi p_{*}(v) \in G(M)$. If $u, v \in G$, then there is a geodesic $\beta$ in $\widetilde{M}$ with $\beta \in[\gamma]$ and $\beta^{\prime}(0)=u$ and $\beta^{\prime}(a)=c v$. Using the fact that $p$ is a geodesic covering, it follows that $p \circ \beta$ has tangent vectors proportional to $p_{*} u$ and $p_{*} v$ at 0 and $a$, respectively. Consequently, $\pi$ takes $p_{*} u$ and $p_{*} v$ to the same image in $G(M)$. Note that $\hat{p}[\gamma]=[p \circ \gamma]$, and $\hat{p}$ is surjective since $p$ is. By construction, $\hat{p} \tilde{\pi}=\pi p_{*}$.

If $W$ is open in $G(M)$, then $p_{*}^{-1}\left(\pi^{-1}(W)\right)$ is open in $T^{\prime} \widetilde{M}$ since $\pi$ and $p_{*}$ are continuous. Thus $\tilde{\pi}\left(p_{*}^{-1}\left(\pi^{-1}(W)\right)\right)$ is open in $G(\widetilde{M})$ because $\tilde{\pi}$ is an open map by Lemma 3.1 of [3]. On the other hand, $\hat{p}^{-1}(W)=\tilde{\pi}\left(p_{*}^{-1}\left(\pi^{-1}(W)\right)\right)$ which establishes the continuity of $\hat{p}$.

If $U$ is open in $G(\widetilde{M})$, then the continuity of $\tilde{\pi}$ yields $\tilde{\pi}^{-1}(U)$ open in $T^{\prime} \widetilde{M}$. Both $p_{*}$ and $\pi$ are open, so we find that $\hat{p}(U)=\pi\left(p_{*}\left(\tilde{\pi}^{-1}(U)\right)\right)$ is open in $G(M)$. Therefore $\hat{p}$ is an open map.

Lemma 3.4 If $M$ is disprisoning, unitrace, or nonreturning, then so is $\widetilde{M}$, respectively. 
Proof: If a geodesic in $\widetilde{M}$ has one end imprisoned in a compact set, so does its projection in $M$. Thus if $\widetilde{M}$ is not disprisoning, then neither is $M$. Similarly, if $\widetilde{M}$ is not unitrace or nonreturning, neither is $M$.

Proposition 3.5 If $M$ is pseudoconvex and nonreturning, then so is $\widetilde{M}$.

Proof: It suffices to show that $\widetilde{M}$ is pseudoconvex. Fix an auxiliary complete Riemannian (positive definite) metric tensor $h$ on $M$ and lift it to $\tilde{h}$ on $\widetilde{M}$. Observe that $p$ is also a geodesic covering for $\tilde{h}$ and $h$ by construction. It follows that $\tilde{h}$ is complete. If $\gamma:(a, b) \rightarrow \widetilde{M}$ is a geodesic of $(\widetilde{M}, \widetilde{\nabla})$, then its $\tilde{h}$-length is equal to the $h$-length of the image $p \circ \gamma$ in $M$. Assume that $\widetilde{M}$ fails to be $\widetilde{\nabla}$-pseudoconvex because there are geodesic segments with endpoints in the compact set $K \subseteq \widetilde{M}$ which fail to be contained in any compact set of $\widetilde{M}$. The continuity of $p$ yields that the image $p(K)$ is a compact set in $M$. Since $M$ is $\nabla$-pseudoconvex, there is a compact set $H$ of $M$ such that any $\nabla$-geodesic of $M$ with endpoints in $p(K)$ must remain in $H$. Cover $H$ with a finite number of $\nabla$-convex normal neighborhoods having compact closure such that any $\nabla$-geodesic that leaves one of these neighborhoods fails to return. For each such neighborhood, there is a maximal $h$-length for each $\nabla$-geodesic segment lying in that neighborhood. Clearly, there is a real number $L$ such that each $\nabla$-geodesic segment which lies in $H$ has $h$-length less than $L$. Now choose any $\widetilde{\nabla}$-geodesic segment $\gamma_{1}$ with $\tilde{h}$-length greater than $L$ and with endpoints in $K$. (Such a $\widetilde{\nabla}$-geodesic segment exists since $\tilde{h}$ is complete and no compact set contains all the $\widetilde{\nabla}$-geodesic segments with endpoints in $K$.) Note that $p \circ \gamma_{1}$ is a $\nabla$-geodesic segment in $M$ which lies in $H$ and has $h$-length greater than $L$; but this contradicts the definition of $L$.

Using this together with Corollary 5.6 of [3], we immediately obtain

Corollary 3.6 If $M$ is nonreturning and $G(M)$ is a manifold, then $G(\widetilde{M})$ is also a manifold.

The next example shows that one may not weaken the assumption that $(M, \nabla)$ is nonreturning to unitrace. It also shows shows that for $(M, \nabla)$ unitrace, $G(M)$ Hausdorff is not equivalent to $(M, \nabla)$ being pseudoconvex.

Example 3.7 Let $M_{0}$ be the real projective plane $\mathbb{P}^{2}$ with the usual elliptic metric and connection. If $M$ is $M_{0}$ less a point, then it is not hard to show that $G(M)=G\left(M_{0}\right)=\mathbb{P}^{2}$ is a manifold and $(M, \nabla)$ is unitrace but not 
pseudoconvex. The universal cover $(\widetilde{M}, \widetilde{\nabla})$ is $S^{2}$ less two antipodal points with the usual connection. Since $M$ is $\mathbb{P}^{2}$ less a point, one may select a geodesic $\gamma$ which is not closed. There are two geodesics $\tilde{\gamma}_{1}$ and $\tilde{\gamma}_{2}$ of $(\widetilde{M}, \widetilde{\nabla})$ lying over $\gamma$ and one may construct a sequence of closed geodesics of $(\widetilde{M}, \widetilde{\nabla})$, the images of which have a Hausdorff closed limit equal to the union of the images of $\tilde{\gamma}_{1}$ and $\tilde{\gamma}_{2}$. Thus $G(M)$ is Hausdorff but $G(\widetilde{M})$ is not.

Proposition 3.8 Assume that $p$ is a finite covering. Then $\widetilde{M}$ is pseudoconvex if and only if $M$ is.

Proof: First assume that $(\widetilde{M}, \widetilde{\nabla})$ is pseudoconvex and let $K$ be a compact set in $M$. Since $p$ is a finite cover, $p^{-1}(K)$ is also compact. Let $\widetilde{H}$ be a compact set in $\widetilde{M}$ such that any $\widetilde{\nabla}$-geodesic segment with endpoints in $p^{-1}(K)$ lies in $\widetilde{H}$. Each $\nabla$-geodesic segment in $M$ with endpoints in $K$ must lift to a $\widetilde{\nabla}$-geodesic segment with endpoints in $p^{-1}(K)$, hence the lift must lie in $\widetilde{H}$. It follows that $(M, \nabla)$ is pseudoconvex because each $\nabla$-geodesic with endpoints in $K$ must lie in the compact set $p(\widetilde{H})$.

Conversely, assume that $(M, \nabla)$ is pseudoconvex and let $\widetilde{K}$ be a compact set in $\widetilde{M}$. Since $p(\widetilde{K})$ is compact, there is a compact set $H$ in $M$ such that any $\nabla$-geodesic segment with endpoints in $p(\widetilde{K})$ lies in $H$. Because $p$ is a finite covering, $p^{-1}(H)$ is a compact set. Now, each $\widetilde{\nabla}$-geodesic segment with endpoints in $\widetilde{K}$ must lie in $p^{-1}(H)$ because it projects to a $\nabla$-geodesic segment with endpoints in $p(\widetilde{K})$ and lying in $H$.

\section{Connectedness}

It will be convenient to modify the definition of sky as used by Low $[8,9]$.

Definition 4.1 For any point $x \in M$, the set $X$ of all geodesics through $x$ is called the sky of $x$.

We shall follow this upper and lower case convention to denote skies.

Theorem 4.2 If $(M, \nabla)$ is unitrace and $G(M)$ is Hausdorff, then $M$ is geodesically connected.

Proof: First, we observe that $G(M)$ is a manifold by Proposition 5.1 of [3]. It is easy to see that the sky of a point $x \in M$ gives a subset $X$ of $G(M)$ that is diffeomorphic to $\mathbb{P}^{n-1}$. 
Because $M$ has a linear connection, $x$ has a convex normal neighborhood. Furthermore, since $M$ is unitrace, there is such a neighborhood which no geodesic through $x$ intersects in more than one connected component. Let $y$ be any point in this neighborhood. Then there is a unique geodesic segment inside the neighborhood connecting $x$ to $y$, and no other geodesic can connect $x$ to $y$ (by the unitrace condition). Hence $X$ intersects $Y$ in a single point.

Now, $X$ and $Y$ are smooth embedded copies of $\mathbb{P}^{n-1}$ in $G(M)$, and under smooth deformations the intersection number is constant modulo 2. But for any $z \in M, Y$ can be smoothly deformed into $Z$ (take a smooth curve in $M$ from $y$ to $z$, and the corresponding skies in $G(M)$ for each point of this curve). Hence $X$ intersects $Z$ in a set with an odd number of elements, and there is always at least one geodesic from $x$ to $z$.

Finally, since $x$ was arbitrary, any two points in $M$ can be connected by a geodesic.

The space $(\widetilde{M}, \widetilde{\nabla})$ of Example 3.7 is unitrace and geodesically connected, but $G(\widetilde{M})$ is not Hausdorff. Thus, for unitrace spaces $M$, the Hausdorff condition on $G(M)$ is sufficient but not necessary for geodesic connectedness of $M$.

The preceding theorem is a peer of Proposition 3.5 of [3], in that there are unitrace spaces with conjugate points (e.g., a plane with a bump), and there are spaces with no conjugate points which are not unitrace (e.g., a cone less its vertex).

One can study the geometry of $(M, \nabla)$ via the topology of $G(M)$. As an example, let $M$ be an open, convex subset of $\mathbb{R}^{n}$ with any linear connection $\nabla$ whose geodesics are the intersections of the usual straight lines with $M$.

Proposition 4.3 For any such $M, G^{+}(M) \cong T S^{n-1}$ whence $G(M)$ is diffeomorphic to an $(n-1)$-plane bundle over $\mathbb{P}^{n-1}$.

Proof: Choose a point $x_{0}$ in $M$ and a Euclidean sphere $S$ centered at $x_{0}$ of a sufficiently small radius so that it is entirely contained in $M$. Let $\gamma$ be an oriented geodesic. Euclidean parallel translate $\gamma$ to $x_{0}$ and let $x$ be the point of intersection of the translation with $S$ in the positive direction. Regarding $T_{x} S$ as embedded in $\mathbb{R}^{n}$, let $P_{x}$ denote the Euclidean orthogonal projection of $M$ onto $T_{x} S$. Extending $\gamma$ if necessary, $\gamma$ intersects $P_{x}$ in a unique point $y$. Now identify $\gamma$ with $(x, y) \in T S$ and note that $P_{x} \cong \mathbb{R}^{n-1}$.

As a specific case, using the Cayley-Klein model of hyperbolic $n$-space $\mathbb{H}^{n}$ we have 
Corollary 4.4 For any standard pseudoeuclidean $\mathbb{R}^{n}, G^{+}\left(\mathbb{H}^{n}\right) \cong G^{+}\left(\mathbb{R}^{n}\right) \cong$ $T S^{n-1}$ whence $G\left(\mathbb{H}^{n}\right) \cong G\left(\mathbb{R}^{n}\right)$ as vector bundles over $\mathbb{P}^{n-1}$.

Geodesic connectedness is a typical example of a geometrically interesting property. The spaces of the preceding example are geodesically connected, as are any which are sufficiently like them. In particular,

Theorem 4.5 If $(M, \nabla)$ has $G^{+}(M) \cong T S^{n-1}$ with skies corresponding to sections over $S^{n-1}$, then it is geodesically connected.

Proof: For brevity, let us denote by $B$ the space $G(M)$ regarded as an $(n-1)$-plane bundle over $\mathbb{P}^{n-1}$. Now recall the Stiefel-Whitney classes of a vector bundle; e.g., 10]. We shall only be concerned with the top class $w_{n-1}(B)$ which is an element of $H^{n-1}\left(\mathbb{P}^{n-1}\right)=\mathbb{Z}_{2}$, the integers modulo 2 . A standard theorem in obstruction theory is that if $w_{n-1} \neq 0$, then $B$ has no nonvanishing sections (if it did, one could split off a line bundle). We show $w_{n-1}(B) \neq 0$.

There are two cases. When $n-1$ is even, $w_{n-1}(B)=w_{n-1}\left(T P^{n-1}\right) \neq$ 0 because $-I$ is homotopic to $I$ as elements of $G L_{n-1}$ and as maps of $S^{n-1}$. When $n-1$ is odd, $w_{n-1}(B) \neq w_{n-1}\left(T P^{n-1}\right)=0$ because $-I$ is not homotopic to $I$ as elements of $G L_{n-1}$ and as maps of $S^{n-1}$. [Indeed, note that in the first case $\operatorname{deg}(-I)=0$ on $S^{n-1}$ and in the second $\operatorname{deg}(-I)=-1$.]

Thus $w_{n-1}(B) \neq 0$ in both cases, so $B$ has no nonvanishing sections, so $M$ is geodesically connected.

In particular, we obtain a new proof of a venerable result.

Corollary 4.6 $\mathbb{H}^{n}$ and any standard pseudoeuclidean $\mathbb{R}^{n}$ are geodesically connected.

More generally, the proof of Theorem 4.5 applies to any $(M, \nabla)$ such that $G^{+}(M)$ is homeomorphic to $T S^{n-1}$ with the skies of points in $M$ corresponding to the sections of $T S^{n-1}$ over $S^{n-1}$. Thus we introduce

Definition 4.7 Let $(E, p, S)$ be a vector bundle. We say $G(M)$ is a sky bundle over $S$, if there is a homeomorphism $F: G(M) \rightarrow E$ such that for each $x \in M$ the map $\varphi_{x}=p(F \mid X): X \rightarrow S$ is a bijection.

Notice that if $G(M)$ is an $E$-sky bundle over $S$, then the skies of $M$ correspond to sections of the vector bundle $(E, p, S)$. For each $[\gamma] \in X$ one has that $F[\gamma]$ is an element in the fiber over $p(F[\gamma])$. Let $h_{x}: S \rightarrow E$ be the section corresponding to $x \in M$. Thus, we have that $h_{x}(s)=F\left(\varphi_{x}^{-1}(s)\right)$. Let $x$ and $y$ denote distinct points of $M$. Then, $h_{x}-h_{y}$ vanishes at some $s_{0} \in S$ if and only if there is a geodesic from $x$ to $y$ in $M$. 
Lemma 4.8 Let $G(M)$ be a sky bundle $(E, p, S)$. If $w_{n-1}(E) \neq 0$, then $M$ is geodesically connected.

Proof: Given any two points $x, y \in M$, let $h_{x}$ and $h_{y}$ be the induced sections. Then $w_{n-1}(E) \neq 0$ implies that there is some point $s_{0} \in S$ with $h_{x}\left(s_{0}\right)-h_{y}\left(s_{0}\right)=0$.

Let $B^{2 n-2}=T S^{n-1} / \sim$ be the identification described earlier. We regard $T S^{n-1}$ as a subset of $\mathbb{R}^{2 n}$ with coordinates $(p, v)$ for $p$ a (Euclidean) unit vector and $v$ a vector (Euclidean) orthogonal to $p$. We identify $(p, v) \sim$ $(p,-v)$ to get the bundle $B^{2 n-2}$. The base space of the vector bundle $B^{2 n-2}$ is $\mathbb{P}^{n-1}$ and the fibers are copies of $\mathbb{R}^{n-1}$.

Corollary 4.9 Let $G(M)$ be a $B^{2 n-2}$-sky bundle over $\mathbb{P}^{n-1}$. Then $M$ is geodesically connected.

Let $F^{+}: G^{+}(M) \rightarrow T S^{n-1}$ be a homeomorphism onto a tubular neighborhood of the zero section and use the above notation. Assume that for each pair of geodesics $\gamma^{+}$and $\gamma^{-}$corresponding to the same geodesic with opposite orientations one has $F^{+}\left(\gamma^{+}\right)=(p, v)$ if and only if $F^{+}\left(\gamma^{-}\right)=(-p, v)$. Then there is an induced map $F: G(M) \rightarrow B^{2 n-2}$ which expresses $G(M)$ as a sky bundle over $\mathbb{P}^{n-1}$. Thus, $M$ is geodesically connected.

We shall now apply this to Hadamard manifolds. First we generalize a definition from [曰, p.2].

Definition 4.10 A manifold with linear connection $(M, \nabla)$ has a pole @ if the exponential map $\exp _{@}: T_{@} M \rightarrow M$ is a diffeomorphism. If every point of $M$ is a pole, we call it a pseudohadamard manifold.

Pseudohadamard manifolds share an essential feature of Hadamard manifolds, as we recall 4 , p. 1].

Definition 4.11 We say that $(M, g)$ is a Hadamard manifold if it is a simply connected, complete Riemannian (positive-definite) manifold of nonpositive sectional curvature.

Greene and $\mathrm{Wu}$ [ [⿶凵 refer to Hadamard manifolds as Cartan-Hadamard (CH) manifolds. The universal cover of a complete Riemannian manifold of nonpositive sectional curvature is a Hadamard manifold. In a Hadamard manifold, the exponential map is a diffeomorphism at each point. Thus, two distinct points of a Hadamard or pseudohadamard manifold are joined by exactly one geodesic. 
Lemma 4.12 A pseudohadamard manifold is nonreturning.

Proof: As noted above, in a pseudohadamard manifold every two points are joined by exactly one geodesic segment. Therefore once a geodesic leaves a convex normal neighborhood it cannot return.

Proposition 4.13 If $(M, \nabla)$ is a pseudohadamard manifold, then it is pseudoconvex and $G(M)$ is a manifold.

Proof: Since $M$ is nonreturning, by Corollary 5.6 of [3] it is sufficient to show that $M$ is $\nabla$-pseudoconvex. First, we note that $M \cong \mathbb{R}^{n}$ and $T M \cong M \times \mathbb{R}^{n}$. We shall regard each $\exp _{x}: \mathbb{R}^{n} \rightarrow M$. Let $K \subseteq M$ be compact and $x \in K$. Then $\exp _{x}^{-1}(K) \subseteq \mathbb{R}^{n}$ is compact. Let $C_{x}=\{\lambda u ; 0 \leq$ $\lambda \leq 1$ and $\left.u \in \exp _{x}^{-1}(K)\right\}$ be the closed cone on $\exp _{x}^{-1}(K)$, so it is also compact. Observe that $H=\bigcup_{x \in K} \exp _{x}\left(C_{x}\right)$ is the $\nabla$-geodesic hull of $K$.

We consider the usual metric on $\mathbb{R}^{n}$ and choose any homeomorphism $M \cong \mathbb{R}^{n}$ to induce a metric on $M$. Let $\delta(x)=\operatorname{diam}\left(\exp _{x}\left(C_{x}\right)\right)$. Observe that $x \mapsto C_{x}$ is a continuous function from $K$ to the set of compact sets in $\mathbb{R}^{n}$ (e.g., with respect to the Hausdorff metric). It follows that $\delta$ is a continuous function $K \rightarrow \mathbb{R}$. Thus it is bounded above on $K$, say by $b$. Then $\{y \in M ; d(y, K) \leq b\}$ is a compact set in $M$ which contains $H$, so $M$ is $\nabla$-pseudoconvex.

For the rest of this paper $(M, g)$ will denote a Hadamard manifold. Let a geodesic triangle lying in $(M, g)$ have sides $c_{1}, c_{2}$ and $c_{3}$ of lengths $a_{1}, a_{2}$, and $a_{3}$, respectively. Assume the angle across from the side of length $a_{3}$ has measure $\alpha_{3}$. For Hadamard manifolds one has the following cosine inequality:

$$
a_{3}^{2} \geq a_{1}^{2}+a_{2}^{2}-2 a_{1} a_{2} \cos \left(\alpha_{3}\right) .
$$

This is known as the first law of cosines; see [1], p. 7 .

Let $p$ be a fixed point of the Hadamard manifold $(M, g)$ and let $c: \mathbb{R}^{1} \rightarrow$ $M$ be a geodesic. Assume $q$ is a closest point to to $p$ on the image of $c$. Notice that if $c_{1}:\left[0, a_{1}\right] \rightarrow M$ is the unique unit speed geodesic from $p$ to $q$, then $c_{1}$ is orthogonal to $c$ at $q$. If $c_{3}:\left[0, a_{3}\right] \rightarrow M$ is a unit speed geodesic from $p$ to some point $r(r \neq q)$ of $c$, then the above inequality shows that $a_{3}>a_{1}$. Hence, $p$ has a unique closest point $q$ on $c$. The point $q$ is called the foot of $p$ on $c$.

Fixing $p$, let $S^{n-1}=\left\{X \in T_{p} M: g(X, X)=1\right\}$. We now define a smooth map $F: T S^{n-1} \rightarrow G^{+}(M)$ for $(M, g)$ as follows. Let $X \in S^{n-1}$ and $Y \in T_{p} M$ with $g(X, Y)=0$. Then $F(X, Y)$ is the geodesic with initial point 
$\exp _{p}(Y)$ and initial direction the parallel transport of $X$ along the geodesic $c_{1}(t)=\exp _{p}(t Y)$. The uniqueness of feet for Hadamard manifolds mentioned above yields that $F$ is a diffeomorphism. Thus, we have established the following result.

Proposition 4.14 If $(M, g)$ is an n-dimensional Hadamard manifold, then $G^{+}(M)$ is diffeomorphic to $T S^{n-1}$.

Once again, we obtain a proof that $\mathbb{R}^{n}$ and $\mathbb{H}^{n}$ are geodesically connected.

Acknowledgement The authors would like to thank the referee for some helpful comments.

\section{References}

[1] W. Ballmann, M. Gromov, and V. Schroeder, Manifolds of Nonpositive Curvature. Boston: Birkäuser, 1985.

[2] J. K. Beem and P. E. Parker, Pseudoconvexity and geodesic connectedness, Ann. Mat. Pura Appl. 155 (1989) 137-142.

[3] J. K. Beem and P.E. Parker, The space of geodesics, Geom. Dedicata 38 (1991) 87-99.

[4] R. E. Greene and H. Wu, Function Theory on Manifolds Which Possess a Pole. LNM 699. New York: Springer-Verlag, 1979.

[5] S. W. Hawking and G. F. R. Ellis, The Large Scale Structure of Spacetime. Cambridge: U.P., 1973.

[6] S. Kobayashi, Transformation Groups in Differential Geometry. Ergeb. Math. Grenz. 70. New York: Springer-Verlag, 1972.

[7] S. Kobayashi and K. Nomizu, Foundations of Differential Geometry, vol. I. New York: Wiley Interscience, 1963.

[8] R. J. Low, The geometry of the space of null geodesics, J. Math. Phys. 30 (1989) 809-811.

[9] R. J. Low, Spaces of causal paths and naked singularities, Class. Quant. Gravity 7 (1990) 943-954.

[10] J. W. Milnor and J.D. Stasheff, Characteristic Classes. Ann. Math. Studies 76. Princeton: U.P., 1974. 
[11] R. S. Palais, A Global Formulation of the Lie Theory of Transformation Groups. Memoirs 22. Providence: AMS, 1957.

[12] P. E. Parker, Spaces of geodesics, to appear in Aportaciones Matemáticas, Serie: Notas de Investigación No. 8, ed. L. Del Riego. pp.61-73. 